\title{
Comparison of Pain Scores between Bamboo and Stainless Steel Finger Traps: An Experimental Study
}

\author{
Thanachai Thongtanworapat, M.D., Porames Suwanno, M.D., Sittichoke Anuntaseree, M.D.
}

Department of Orthopaedic Surgery and Physical Medicine, Faculty of Medicine, Prince of Songkla University, Hat Yai, Songkhla 90110, Thailand.

Received 21 December 2018 • Revised 9 March 2019 • Accepted 19 March 2019 • Published online 3 May 2019

\section{Abstract:}

Objective: The Chinese finger trap is a device used to aid in reduction of fractures, especially in distal end of radius fractures. The stainless steel finger trap is widely used but often causes fingers pain. We adapted a bamboo finger trap to reduce pain and also provide a lower cost alternative.

Material and Methods: This was a cross-over study in healthy volunteers comparing bamboo and stainless-steel finger traps. Each participant underwent two tests, one with the stainless steel finger trap and another with the bamboo finger trap. For each trial, the participant lay supine on a bed, and the finger trap was attached to the index and middle fingers of the participant, with the arm suspended by a metal loop at the top of the trap to the elbow level of the patient on the bed. Weights were incrementally added to a weight bag suspended by a strap over the patient's upper arm at one pound per minute until a maximum weight of 20 lbs. and the participant was asked to rate the degree of discomfort with the increasing weights using a visual analogue score (VAS)

Results: Thirty volunteers were tested, all of whom were tested with both the bamboo and stainless steel traction devices. One patient developed a superficial skin injury while the stainless steel device was being applied. Overall, the bamboo finger trap group had lower VAS scores than the stainless steel group in the first fifteen minutes ( $p$-value< 0.001).

Conclusion: The bamboo finger trap is an effective alternative to the stainless steel finger trap, causing less pain.

Keywords: bamboo, Chinese finger trap, pain score, stainless

Contact: Porames Suwanno, M.D.

Department of Orthopaedic Surgery and Physical Medicine,

Faculty of Medicine, Prince of Songkla University, Hat Yai, Songkhla

90110, Thailand.

E-mail: ake23488@gmail.com
J Health Sci Med Res 2019;37(2):145-150 doi: 10.31584/jhsmr.201949 www.jhsmr.org 


\section{Introduction}

The finger trap is a medical device that is widely used in patients with bone and joint diseases, and especially for treatment of distal radius fractures. ${ }^{1}$ The method of use is to suspend the patient's arm using a finger trap and to disimpact the fracture by applying weight to the elbow followed by reduction and immobilization. A finger trap can also be used in some types of hand and wrist surgery such as wrist arthroscopy.

The distal radius fracture is common in the emergency department, with up to 640,000 people suffering from this injury per year in the United States of America, about $3.0 \%$ of all injuries to the upper limb. ${ }^{2,3}$ The incidence in Songklanakarind Hospital, the major tertiary care center in southern Thailand, during the 5year period 2007-2012 averaged 91 people per year. The distal radius fracture is a serious injury, resulting in pain and often loss of job and income. Initial realignment or reduction of the fracture is the standard treatment. The reduction can be done manually or through mechanical traction using a finger trap device; studies have found no differences in anatomical outcomes between the two methods, but mechanical traction involved less pain during the reduction and fewer instances of long-term neurological impairment. ${ }^{4,5}$

The commonly used commercial finger traps are made from stainless and thus have a long life, but they also have high contact pressure on the skin of the fingers, leading to suffering in the patient from pain or finger injury. ${ }^{6}$ adapted a bamboo finger trap (Figure 1) made from dry young bamboo trunk to solve this problem. In a previously study, palm leaf finger traps were mechanically tested by an Introns 8875 machine with Moore's construct in a cadaveric study that showed it can be used to pull the finger $^{7}$ There have been, however, no clinical studies. This led do research with volunteer in our study.
The purpose of this study was to compare pain scores between the bamboo finger trap and the stainless steel finger trap. The hypothesis was that there would be differences in associated finger pain scores between two types of devices in volunteers.

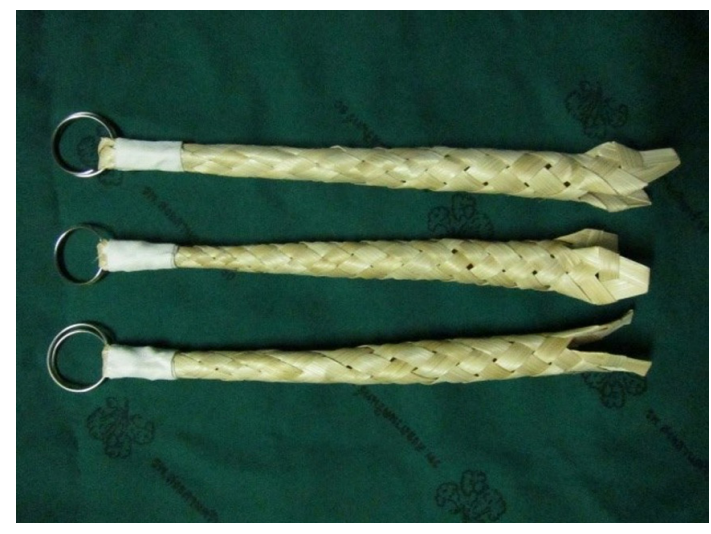

Figure 1 Bamboo-trunk finger trap

\section{Material and Methods}

\section{Study population}

The subjects of this study were healthy volunteers aged 18-65 years old. Volunteers with a history of upper extremity problems including injury, surgery, skin abnormalities or sensory impairment were excluded. All volunteers provided informed consent before the trials began. The study was approved by the Ethics Committee of the Faculty of Medicine, Prince of Songkla University.

\section{Testing}

The volunteer was instructed to lie flat on a bed eyes closed. A research assistant opened an envelope to see which kind of finger trap (bamboo or stainless steel) was indicated, and then applied the trap to the index and middle fingers, after which the device was covered with a cloth bag so that neither the volunteer nor the 
assessor could see which type of finger trap was being used. The finger trap was suspended above the bed with a hook attached to the device, and to the side so that the volunteer's arm was suspended vertically by a weight hook. A weight bag was placed over the volunteer's upper arm, and the assessor began adding weights to the bag at a rate of 1 pound per minute until 20 pounds had been added (Figure 2). If there was some kind of equipment damage the test was stopped. The volunteer was asked to rate their finger pain using a visual analog score (VAS). The trial was stopped if the VAS became more than 8 points. The pain scores were requested in one-minute intervals along with the weight increases. All volunteers were tested with the other type of finger trap at a second session two weeks after the first session.

\section{Evaluation}

The test would be stopped when the test was completed, i.e. twenty pounds had been added to the weight bag, or the volunteer indicated severe pain (VAS> 8 points), or they felt too much discomfort to continue

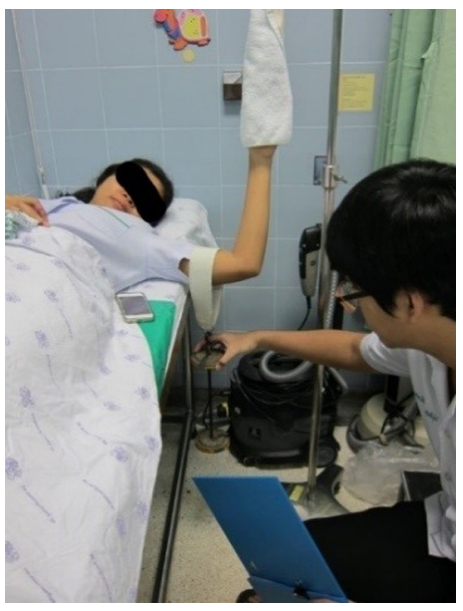

Figure 2 Testing and measurement method the test, or if the finger trap became damaged or one or both of the fingers became dislodged from the trap. The assessor monitored the fingers carefully to ensure there were no finger injuries.

\section{Statistical analysis}

Differences in categorical variables were analyzed by chi-squared test and continuous variables were analyzed by unpaired t-test. Data are presented as mean \pm standard deviation (S.D.), ratio, or percentage. A p-value of less than 0.05 was regarded as statistically significant.

\section{Results}

Thirty volunteers were tested, 13 men 17 women. The mean age of the volunteers was 30.3 years, and the mean body mass index was $24.3 \mathrm{~kg} / \mathrm{m}^{2}$. Twenty-six were right-hand dominant and 4 were left-hand dominant (Table 1). All volunteers tested both types of finger trap two weeks apart. Overall, the bamboo finger trap group had lower VAS pain scores than the stainless steel finger trap

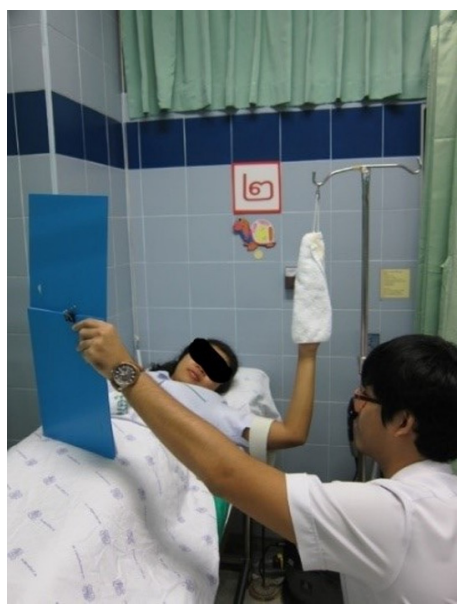


group for the first fifteen minutes ( $p$-value<0.001). After fifteen minutes the VAS pain scores were not significantly different between the two groups (Table 2). All of both the stainless steel and bamboo finger traps were able to maintain their hold on all fingers in all subjects. None of finger traps showed any damage during the study. One volunteer developed a superficial skin injury while a stainless steel finger trap was applied (Figure 3), but the wound healed in a few day. No subjects recorded a pain score greater than $8 / 10$ or asked for the test to be stopped.

Table 1 Demographic data of the volunteers

\begin{tabular}{ll}
\hline Demographic characteristic & $\begin{array}{l}\text { Volunteers } \\
\text { Number }(\%)\end{array}$ \\
\hline Sex & \\
Male & $13(43.3)$ \\
Female & $17(56.7)$ \\
Age (mean, years) & 30.3 \\
BMl $\left(\mathrm{kg} / \mathrm{m}^{2}\right)$ & 24.2 \\
Dominant hand & \\
Right & $26(86.7)$ \\
Left & $4(13.3)$ \\
\hline
\end{tabular}

$\mathrm{BMI}=$ body mass index
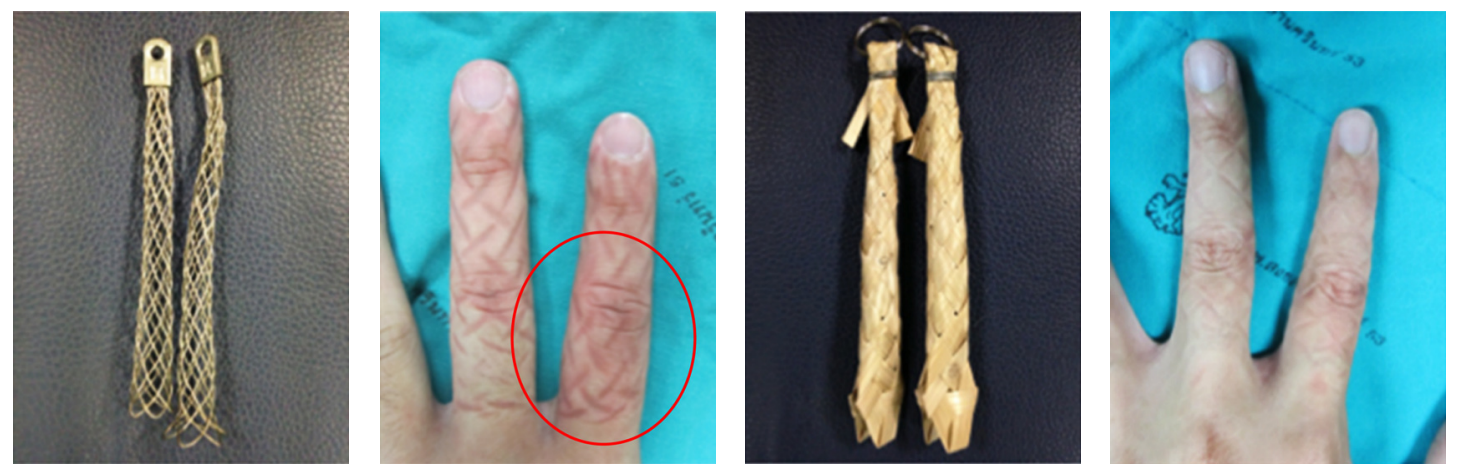

Figure $3 \mathrm{~A}$ complication from the metal finger is superficial skin ablation 


\section{Discussion}

The finger trap is a useful device to help surgeons manage certain orthopedic problems such as aiding in reduction of distal radius fractures. It is not without drawbacks, however, as if too much weight is applied it can cause severe pain or finger injury.

To date there have been no studies comparing pain severity or finger injuries between various types of finger traps. Polasak et al. ${ }^{7}$ did a study on the durability of palm leaf, stainless steel, and nylon finger traps using a finger model, and found that no type of finger trap failed when the weight used was not more than 20 pounds; when over 60 pounds of weight was applied, both the stainless steel and nylon finger traps failed. They concluded that the palm leaf finger trap works well in normal situations, but they did not test the finger model, and pain severity was not evaluated.

The bamboo finger trap can be used in volunteers without failure or slipping from volunteer's finger with determines quality control of production process and mechanical and cadaveric study prior to test in this study. This study found that bamboo finger trap use resulted in a significantly lower pain score during 15 minutes than the stainless steel traps (Figure 4). This may be due to the trap's construction from thin, flexible and wide sheets of bamboo-trunk material that reduces contact pressure on the skin. No complications such as finger injury or severe pain were observed due to the soft characteristics of bamboo-trunk material. Further studies on the life expectancy and cost-effectiveness of the trap should be done, and also on the clinical factors involved in longer-term trap use.

\section{Conclusion}

The study found that the bamboo-trunk finger trap resulted in lower pain scores and was safe to use without any complications compared to the stainless steel finger trap.

\section{Aknowledgement}

Playable museum Chiang Rai Thailand.

\section{Conflict of interest}

No potential conflict of interest relevant to this article was reported.

\section{Traction Time(Minute) and Pain score (Visual Analog Score )}

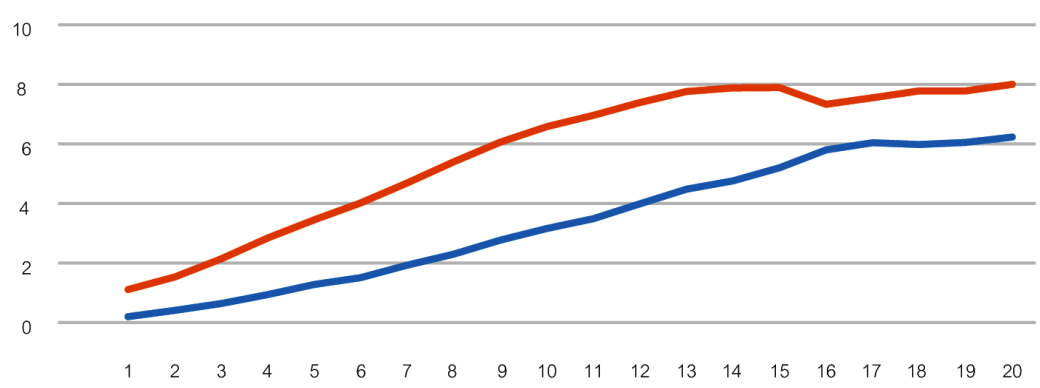

\section{VAS Bamboo - VAS Stainless steel}

Figure 4 Comparing pain scores (mean) and time between the two finger traps 


\section{References}

1. Kongsholm J, Olerud C. Reduction of Colles' fracture without anesthesia using a new dynamic bone alignment system. Injury 1987;18:133-6.

2. Chung KC, Spilson SV. The frequency and epidemiology of hand and forearm fractures in the United States. J Hand Surg Am 2001;26:908-15.

3. Wolfe SW. Distal radius fracture. Green's operative hand surgery. $7^{\text {th }}$ ed. Philadelphia: PA Elsevier; 2017;17:516-31.

4. Earnshaw SA, Aladin A. Closed reduction of Colles fractures: comparison of manual and finger-trap traction: a prospective, randomized study. J Bone Joint Surg Am 2002;84-A:354-8.

5. Handoll HHG, Madhok R. Closed reduction methods for treating distal radial fractures in adults. Cochrane Database Syst Rev 2003;1:1-7.

6. Spurrier E. Pressure exerted by finger traps. J Perioper Pract 2011;21:140-1.

7. Jeeravipoolvarn $\mathrm{P}$, Jeeravipoolvan $\mathrm{B}$, Mahaisavariya B. A comparative study of durability of palm leaf, nylon and stainless steel finger traps. Srinagarid Hosp Med J 1986;2:113-7. 